\title{
SMARTPHONE BASED IN/OUT TICKETING SYSTEMS: A NEW GENERATION OF TICKETING IN PUBLIC TRANSPORT AND ITS PERFORMANCE TESTING
}

\author{
MATTHIAS H. WIRTZ \& JAN KLÄHR \\ Rhein-Main-Verkehrsverbund Servicegesellschaft mbH, Germany
}

\begin{abstract}
AFC-systems with In/Out technology allow the passenger to use public transport and be billed based on their service consumption afterwards. Up to now these systems have utilized smartcards and a regulated access to the pt-network including a Check-In and Check-Out process. For open access transport systems, new smartphone-based AFC-systems are developed offering the comfort of automatic price calculation (pay-as-you-go) while maintaining a gate-free access to the pt-service. We present the differing system characteristics regarding user processes at trip start and end and the data recorded. Furthermore we illustrate that high accuracy of the resulting trip information is important for the revenue and how the accuracy can be measured and proved in the field.

Keywords: public transport, AFC-system, ticketing, smartphone, In/Out System, accuracy, testing, Be-Out.
\end{abstract}

\section{INTRODUCTION}

The majority of AFC-systems (automatic fare collection), which have been introduced worldwide, have implemented a smartcard based CICO (Check-In/Check-Out) system. Public transport users check-in and check-out at the beginning and end of their trips so that the system records the service which has been utilized. In Germany most introduced smartcard AFC-systems only replace paper based tickets without introducing CICO processes, so far [1]. In the service area of the Rhein-Main-Verkehrsverbund (Rhein-Main regional transport association) e.g. the majority of paper based monthly and season ticket types have been replaced by smartcards.

These systems cannot record the service utilized by the user and lack the option for automated price calculation. Therefore they don't alleviate the user from choosing the right ticket type and value. The same holds true for smartphone based AFC-systems in Germany. They do not record the service utilized and replaced mainly the single trip tickets, return tickets and day passes. By now only a few systems in Germany incorporate a CICO process e.g. the Kreisverkehr Schwäbisch Hall transport association [2] or the Stadtwerke Münster.

One important objective for implementing an AFC-system that records the utilized service includes the increased comfort for public transit users by offering an automated price calculation (pay-as-you-go). This can be achieved by introducing gates at the entrance and exists of the public transportation network and implement a CICO process at these gates. For open access interconnected public transportation systems such as those in Germany a technology that supports open access might be a better fit. Therefore different smartphone based AFC-systems which are capable of recording the utilized service while preserving the open access transportation system have been developed recently. Since different processes are used at the start and end of the journey we refer to these systems as smartphone based In/Out-Systems.

In this paper we will present the essential building blocks of such systems and describe their main differences in the user interface and in the data which is recorded. We will illustrate the importance of highly accurate trip data recorded by the system due to their impact on 
revenue and present a method for accuracy measurement. Finally we will present different methods for testing such systems and discuss their advantages and disadvantages.

\section{BASIC SYSTEM ARCHITECTURE}

Smartphone based In/Out AFC-Systems are implemented with different intentions and therefore show distinctive architecture concepts [3]. Nevertheless all systems have some basic components in common which are:

- $\quad$ smartphone app

- $\quad$ backend trip construction module

- backend billing/CRM module

- optional: pt-vehicle or pt-station infrastructure

In the following section we focus on the user interaction with the smartphone app at start and end of the journey and the type of collected data during system usage. The typical approaches are differentiated and discussed.

\subsection{Processes at start and end of the journey}

\subsubsection{Check-In/Check-Out (CICO)}

The Check-In and Check-Out processes are well known from smartcard based AFC-systems which record the service utilized. In contrast to smartcard based systems where the CICO processes is enforced by gates and therefore takes place at a physical device (POS) the CICO process in smartphone based systems is generally not bound to any physical infrastructure and can take place anywhere in vicinity of the public transport stop (pt-stop). Thus they are often referred to as virtual Check-In and virtual Check-Out processes.

Most operational AFC-systems inform the user during the Check-In process which pt-stop the system has selected as the start point of the first trip. This selection can be overridden by the user if necessary. The option for overriding is usually limited to stops within a certain distance (e.g. 500 meters) to the stop selected by the system. The double checking of the start point is at least a small effort for the user but lets him know what is going on and affirms him about the correct selection of the start location.

The Check-Out process is also initiated by the user but with no option of overriding the selected alighting stop. Generally at the end of this process the user is informed about the service consumed and the price of the service.

The oldest representative in Germany with a Check-In and Check-Out process is the Touch\&Travel system [4] of the Deutsche Bahn AG. Other systems implementing a Check-In and Check-Out process are e.g. Fairtiq [5] and Lezzgo [6].

\subsubsection{Be-In/Be-Out (BIBO)}

Processes that do not necessitate direct interaction with the smartphone-app at trip start and end are often referred to as Be-In and Be-Out. Although systems implementing a Be-In or Be-Out process still do need some user interaction but they don't require the user to e.g. terminate the process manually when reaching the last pt-stop of their journey. So these systems alleviate the user substantially from manual Check-In and Check-Out processes are therefore called Be-In and Be-Out.

There are currently no productive smartphone based AFC-systems which provide a Be-In process. In the EILO project funded by the German Federal Ministry of Transport and Digital Infrastructure [7] a Be-In process is developed and tested. When starting the smartphone app 
and initiating the trip recording the user doesn't necessarily need to be at the first pt-stop of the journey but rather on the way to a pt-stop or even at home. The backend processes analyse the data and location information from the smartphone and compare these with the location and trajectory of the pt-vehicles. Only if there is a close correlation between both a corresponding trip is generated. A similar process is tested for the Be-Out process: if the correlation of the trajectory between pt-vehicle and smartphone does no longer exist a Be-Out event is triggered and sent to the smartphone-app. Both processes rely heavily on accurate trajectories of the pt-vehicles.

MotionTags TicketEasy solution [8] in contrast offers a Be-Out functionality which is mainly based on recognizing the actual mode of travel by analysing the data of the inertial sensors of the smartphone. The assumption hereby is that once the user has reached its last pt-stop of his journey the mode of travel changes to walking or walking in combination with other non pt-modes. If such a transition of mode is recognized with a certain degree of probability a Be-Out event is triggered.

Since the sensor data does not follow a standard and is therefore highly vendor and hardware specific it seems to be very demanding to implement a solution which works on almost all smartphone models.

\subsubsection{Assisted Check-Out (ACO)}

The assisted Check-Out adds more comfort to the Check-Out process without completely alleviating the user from the responsibility to supervise the Check-Out process like in Be-Out processes.

The Fairtiq system uses a similar approach as the MotionTag system by identifying the actual mode of travel. Once the system detects a non pt-mode the user receives a reminder of an active trip and a Check-Out request. The final decision still relies on the user and if no action is taken the system keeps on recording the users' movement.

\subsection{Collected data}

For all In/Out Ticketing Systems it is crucial to record the necessary data in a certain quality in order to be able to reconstruct the trips made by the customer.

\subsubsection{Location data provided by the smartphone operating systems}

The most important data source is the location information retrieved from the smartphone app. To the best of our knowledge all productive systems and currently under development utilize the location data provided by the operating system of the smartphone. Modern smartphone operating systems use a combination of several sensors like GNSS, WLAN, BLE or accelerometer in order to provide accurate location information. For a detailed description of smartphone location data and sensor fusing see [9].

\subsubsection{PT-vehicle generated data}

In addition or as a possible replacement for location data provided by the smartphone the pt-vehicles could be used to transmit identifying data. In a simple form this might be static beacon information which allows the receiving smartphone to identify the vehicles in its vicinity. More advanced setups might include transmitting dynamic information which could include the destination of the vehicle and the upcoming stops.

Data transmitted from the pt-vehicles are especially advantageous when the pt-service is located underground or is covered up so that the location service of smartphones can't 
provide reliable location data anymore. It might be advantageous for a Be-Out functionality too since the loss in receiving data is a strong indication for the alighting of the pt-vehicle.

The AFC-system developed by Turnit [10] and the system developed in the federal state funded project esim 2020 [11] both used pt-vehicle generated data. The former system uses Bluetooth Low Energy (BLE) as data transmitting standard and the latter used the WLAN standard.

\subsubsection{PT-Station generated data}

Similar to data transmitted by pt-vehicles data can be transmitted by infrastructure located at pt-stations. Most likely the data will be comprised of static beacon information and will especially be advantages for underground pt-stations and the Be-Out functionality.

\section{PERFORMANCE MEASUREMENT}

\subsection{Measurement of accuracy}

The aspect of system accuracy has different facets in In/Out Ticketing Systems compared to AFC systems which do not record the utilized service. The latter systems depend on the user to specify in advance the service they intend to use. It's the users' responsibility to purchase the proper ticket type and value before starting the trip. This is different for CICO and BIBO as in BIBO systems the responsibility for identifying the utilized service moves from the user to the AFC system.

Even within the family of In/Out Ticketing Systems the aspect of accuracy has different levels. When Check-In and Check-Out processes are implemented the user gets an immediate feedback of the outcome of these processes and could take appropriate action if the feedback is not as expected. If the start and end location of the journey is double checked by the user the post processing could heavily rely on this data.

This is not the case if Be-In and Be-Out processes are implemented. In this case the user does neither assess the data recorded during start and end of the journey nor at any other time. Therefore the room for possible inaccuracy is much bigger and post processing can't rely on any verified data points.

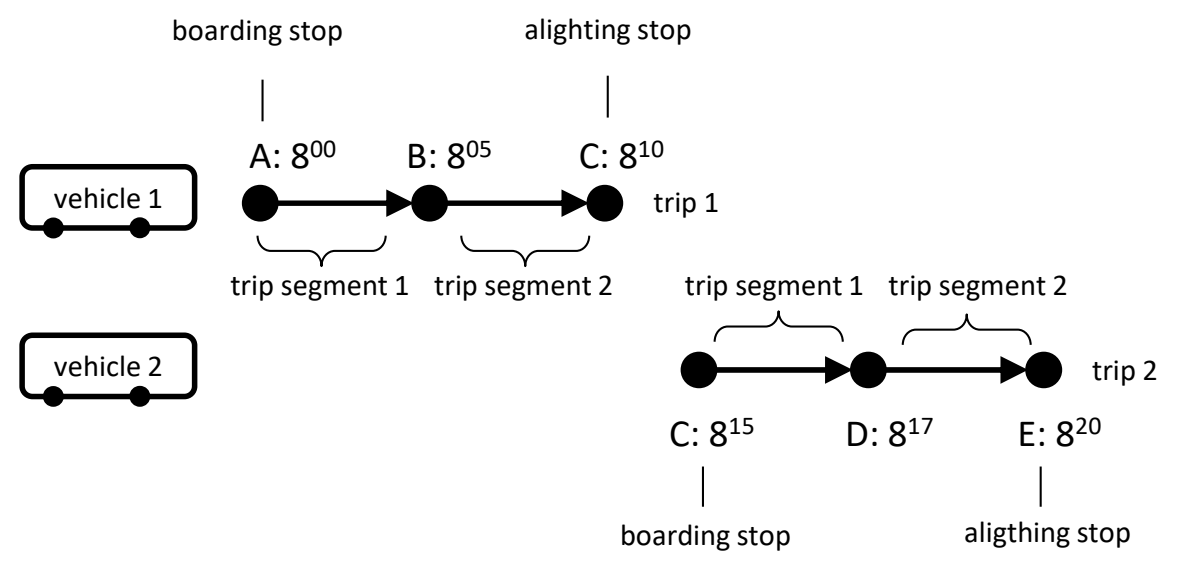

Figure 1: Definition of trip and trip segment. 
In order to prevent misconceptions when defining the accuracy measurement we explain the terms used in a small example given in Fig. 1. First the customer boarded vehicle 1 at stop A and got off the vehicle at stop C. All this comprises the first trip which consists of two trip segments. The second trip started at stop $\mathrm{C}$ and ended at stop $\mathrm{E}$ and was done using vehicle 2 . Since there was a vehicle change at stop $\mathrm{C}$ many pricing schemes would price both trips together on a single ticket. In this case both trips would comprise one journey. Otherwise each trip would be a journey of its own with its own price.

We prefer the segment based accuracy measurement in cases where the system performance from a technical perspective is required. This measurement is completely independent of the pricing schema so that the result of the measurement does not change if the pricing schema is modified. In addition this measurement is able to detect rather small changes in system performance since it is based on the smallest part of each and every trips: the trip segment.

According to Wirtz [12] the trip segment based accuracy $A_{T S}$ is calculated as following:

$$
A_{T S}=\frac{\sum_{U M} T S_{\text {correct }}}{\sum_{U M} T S_{\text {correct }}+\sum_{U M} T S_{\text {fault }}},
$$

with $T S_{\text {correct }}$ as correct recorded trip segment, $T S_{\text {fault }}$ as incorrect recorded trip segment and $U M$ as each smartphone (user medium). If a trip segment has been recorded correctly is determined by:

- the accurate start and end stop of each segment,

- the accurate time of departure and arrival (should be within a predefined time window of the real time),

- and optionally the correct index number of the segment within the trip.

The incorrect recorded trip segments might be distinguished by missing and additional trip segments and weighted differently. In this case $T S_{\text {fault }}$ is calculated as following:

$$
T S_{\text {fault }}=w_{\text {additional }} * T S_{\text {additional }}+w_{\text {missing }} * T S_{\text {missing }}
$$

with $T S_{\text {additional }}$ as trip segment which was not travelled along by the user in reality but was recorded as utilized by the system and $T S_{\text {missing }}$ as trip segment which was travelled but was not recognized by the system. The main ratio behind the different weights for additional and missing trips are the different perceptions of these error types by the users. Additional trip segments might lead to an increase in price which most likely will be complained more intense while missing trip segments might lead to a reduced price and most likely not criticised in the same extend.

A detailed discussion of different methods for accuracy assessment can be found in [12].

\subsection{Measurement of Be-Out effectivity}

If the AFC-system supports a Be-Out or an assisted Check-Out functionality the effectivity of this process can be evaluated too. In the following we will only speak of Be-Out functionality but do encompass herein an assisted Check-Out functionality too.

While the accuracy of the AFC-system is relevant for both the user acceptance and the revenue a Be-Out functionality is mainly relevant for the user experience. Therefore we focus on the impact on user experience of the Be-Out functionality when measuring the effectivity. 
We consider two main characteristics of the Be-Out process affecting the user experience: the number of falsely triggered Be-Outs and the time span between the arrival time at the final stop and the time point when the Be-Out is triggered. The former factor was weighted with $80 \%$ and the latter factor with $20 \%$. The Be-Out effectivity $E f_{\text {BeOut }}$ is calculated as following:

$$
E f_{\text {BeOut }}=0.8 * \frac{n-\sum(T E+T L)}{n}+0.2 * \frac{n-\sum T L-\frac{\sum T T B}{20 \text { minutes }}}{n},
$$

with $T E$ as an Be-Out event that was triggered to early, $T L$ as an Be-Out event that was triggered to late, TTB the time span in minutes between the alighting of the vehicle the following Be-Out event (time to Be-Out) and $n$ being the number of trips. The Be-Out effectivity value margin lies within $-\infty$ and 1 whereas the latter represents the best effectivity and all smaller values represent lower effectivities.

The events early and late Be-Out are defined as following:

- Early Be-Out: a Be-Out is triggered during a trip, while transferring to a connecting trip or waiting to start the first trip for less than 20 minutes.

- Late Be-Out: a Be-Out is not triggered although the final stop had been reached more than 20 minutes ago.

\section{TEST PLANNING}

Test planning has a great impact on the quantity and quality of the generated data for the accuracy assessment. Generally, paid test personal should be used in order to ensure high quality of the documentation during the test trips. There are two main different approaches for organizing the test personal:

- Groups: the test persons are grouped together and all members of a group performing the exact same trips at the same time. One person is leading the group and is responsible for the documentation.

- Individually: each test person performs the test trips individually and documents his trips separately.

Each organization form has its unique advantages and might be the best choice depending on the basic conditions. The main characteristics of both organization forms are shown in Table 1.

In addition to the organization of the test persons the basic test setup plays a key role. The basic test setup is defined by the so-called trip diary. The trip diary documents all trips done including at least the start and end stop of each trip, the departure and arrival time and the used mode of transport. Depending on the functionality of the AFC-system other parameters might be necessary too. If there is e.g. a Be-Out functionality the necessary data describing the Be-Out like the time at which the Be-Out was received has to be recorded too.

The trip diary might be provided in two different settings:

- $\quad$ Preset trip diary: the trips which should be made by the test persons and their order are defined beforehand. The location of each boarding and alighting, the mode and route to use is all defined and prefilled in the trip dairy.

- Free floating trip diary: Only basic guidelines are defined beforehand regarding e.g. a daily time frame during which the test or the geographical area in which the tests 
should be made. Besides these guidelines the test person is free to choose which route to use and where to start and end each trip.

Similar to the organization of the test persons each setting of the test setup has its advantages. The main characteristics of each setup are given in Table 2 .

Table 1: Characteristics of test group organization forms.

\begin{tabular}{|c|c|}
\hline Organization form & Characteristics \\
\hline Group & $\begin{array}{l}\text { - } \text { only group leaders need to be fully trained } \\
\text { - } \quad \text { supervision by group leaders easily possible } \\
\text { reduced effort for processing the trip diaries within each } \\
\text { group due to the uniformity of the trips } \\
\text { individual behaviour superimposed by behaviour of group } \\
\text { leader (lemming effect) } \\
\text { homogenous test setting within each group allowing to } \\
\text { identify influence of smartphone model and operating } \\
\text { system } \\
\text { performance of group depends largely on performance of } \\
\text { group leader }\end{array}$ \\
\hline Individually & $\begin{array}{l}\text { - individual behaviour of all test persons } \\
\text { - } \text { heterogenic data reflecting the real word usage rather } \\
\text { accurate } \\
\text { - all test persons need to be fully trained } \\
\text { - high variance in performance of test person }\end{array}$ \\
\hline
\end{tabular}

Table 2: Characteristics of the general test setup.

\begin{tabular}{|l|l|l|}
\hline General test setup & Characteristics \\
\hline Pre-set trip diary & - & specific test scenarios can be tested (edged cases) \\
& - & better supervision of which pt-service should be tested. \\
& - & $\begin{array}{l}\text { reduced probability of violation of test guidelines } \\
\text { reduced motivation of test person due to lacking }\end{array}$ \\
& - & $\begin{array}{l}\text { self-dependency in planning } \\
\text { disturbances in pt-service might hamper test trips }\end{array}$ \\
\hline $\begin{array}{l}\text { Free floating trip } \\
\text { diary }\end{array}$ & - $\begin{array}{l}\text { high motivation of test persons due to self-dependent trip } \\
\text { planning }\end{array}$ \\
& - $\begin{array}{l}\text { reduced planning effort beforehand since no pre-set trip } \\
\text { diary is necessary } \\
\text { disturbances in pt-service can easily be compensated by } \\
\text { flexible planning } \\
\text { guidelines might be violated especially when reasons are } \\
\text { unknown } \\
\text { bias to use more frequent and/or for the test person more } \\
\text { familiar pt-services }\end{array}$ \\
\hline
\end{tabular}


Besides these characteristics group organization form and test setup the development status of the AFC-system plays an important role when deciding which test setup to use. If the AFC-system is rather in an early development stage it might be the best option to use preset trip diaries and grouped test persons in order to generate large amounts of rather similar data more easily. So variances introduced by the individual behavior of the test person can be kept to a minimum. In addition when using different smartphones in each group their variances can be identified and analyzed in detail. Furthermore this setup allows generating large amounts of test data rather easily while reducing the probability of edge cases and unusual data. This should help streamline the early development stages of the AFC-system where lager and more frequent changes in software are made.

If the AFC-system is in a more matured development stage it might a good option to use preset trip diaries with individual test persons. Thereby the time points at which the trips are done are spread over the desired time window and external factors e.g. the individual behavior of each test person is reflected in the recorded data. In addition scenarios which are known to be hard to handle for the AFC-system can be tested in a precise manner. Finally one should use free floating trip diaries with individual test persons. This setup represents the real world closest and will reveal errors and unusual situations that were not thought of beforehand and therefore not covered by the preset trip diaries.

\section{OUTLOOK}

Smartphone based In/Out AFC-systems seem to have great potential for occasional users who usually don't have season tickets. Especially in open access public transport systems In/Out AFC-systems can provide an automatic price calculation, a pay-as-you-go option. In addition they provide the basis for a more flexible and demand depending price calculation. Since the AFC-system is then responsible for recognizing the trips made correctly there is an increased effort necessary to ensure a correct functionality of the system. The right balance between pursuing to automate all possible processes on the user side and keeping the systems' accuracy performance on a sufficient level seems to be the challenge of the future.

\section{ACKNOWLEDGEMENT}

The work presented is partly based on the results of the project "EILO: Einsteigen und Losfahren" funded by the German Ministry of Transportation and Digital Infrastructure.

\section{REFERENCES}

[1] Janssen, S. \& Loersch, H., e-Ticketing in Germany. Eurotransport, pp. 20-23, 2005.

[2] Kühnel, I., Major advances in eTicketing. Eurotransport, pp. 20-23, 2005.

[3] Narzt, W., Mayerhofer, S., Weichselbaum, O., Haselblöck, S. \& Höfler, N., Bluetooth low energy as enabling technology for be-in/be-out systems. Proceedings of the 2016 13th IEEE Annual Consumer Communications \& Networking Conference, 2016, Las Vegas, USA. DOI: 10.1109/ccnc.2016.7444817.

[4] Deutsche Bahn, A,G., Touch\&Travel-Ein innovatives eTicketing-Verfahren, 2008.

[5] Fairtiq. Online. www.fairtiq.ch/en. Accessed on: 19 May 2018.

[6] Lezzgo. Online. www.lezzgo.ch/index-en.html. Accessed on: 19 May 2018.

[7] Huber, M. \& Reher, R., In-Out-Lösungen revolutionieren den Ticketkauf im ÖPNV. ETR_Eisenbahntechnische Rundschau, 2017(6), pp. 31-35, 2017.

[8] Motion-Tag. Online. motion-tag.com/ticketing. Accessed on: 19 May 2018.

[9] von Watzdorf, S. \& Michahelles, F., Accuracy of positioning data on smartphones. Proceedings of the 3rd International Workshop on Location and the Web, pp. 2:1-2:4, 2010. DOI: 10.1145/1899662.1899664. 
[10] Turnit. Online. turnit.com/product/bibo/. Accessed on: 19 May 2018.

[11] Ringat, K. \& Puzicha, J., Auf dem Weg zum Ticket der Zukunft. Der Nahverkehr, 2016(12), pp. 31-35, 2016.

[12] Wirtz, M., Erfassungsgenauigkeit von smartphone basierten BIBO-EFM-Systemen. Proceedings of the Nahverkehrs-Tage, 2017, pp. 111-123, 2017. 